\title{
Iterative Scattering by Two PEMC Elliptic Cylinders
}

\author{
A.-K. Hamid \\ Electrical and Computer Engineering Department, University of Sharjah, Sharjah, UAE \\ *corresponding author, E-mail: akhamidesharjah.ac.ae
}

\begin{abstract}
Iterative procedure is implemented to derive rigorous solution to the problem of plane electromagnetic wave scattering by couple of perfect electromagnetic conducting (PEMC) elliptic cylinders due co and cross polarized scattered fields among cylinders. The translation addition theorem for Mathieu functions is enforced to compute the higher order scattered fields by single PEMC elliptic cylinder in terms of the other elliptic cylinder coordination system to impose the boundary conditions. The kth co and cross polarized scattered field coefficient expressions are extracted by iteration procedure without using matrix inversion.
\end{abstract}

\section{INTRODUCTION}

There are many practical solutions that rely on the scattering by infinite elliptic cylinders such as studying the scattering by irregular cylinder simulated by elliptic cylinders, also verifying the numerical and approximate technique solution results.

Translation addition theorem for Mathieu functions is forced to solve analytically the problem of scattering by a system of PEMC elliptic cylinders in order to apply the boundary conditions. As the number of elliptic cylinders increase, additional memory and time are required to invert the coupling system matrix. Further, for some given cylinder sizes, separation distances, material constants and angles of incidence are hard to achieve because of the related ill-condition system matrices.

In this paper, an iterative procedure is presented to the scattering by couple of PEMC elliptic cylinders. The procedure needs to solve the scattered co/cross polarized fields by each PEMC cylinder as it acts as incoming field on the other cylinder. The initial scattered fields may be obtained due to the initial incoming field only. The second order scattered fields may be obtained by exciting every cylinder by the initial co/ cross polarized scattered fields.

There are available solutions to the multiple interaction as by conducting and dielectric elliptic cylinders as in [1-3], circular cylinders [4-6], spheres [7] and spheroids [8] using different techniques.

We implement in the current paper an iterative procedure for couple of conducting elliptic cylinders to develop the solution for scattering by PEMC elliptic cylinders [3]. The proposed solution will also present the scattering from perfectly electric conducting (PEC) cylinders by making the PEMC admittance goes to infinity on cylinders surface while reducing to the scattering from perfectly magnetic conducting (PMC) cylinders by letting PEMC the admittance goes to zero. Expressions are obtained in the next section, without using matrix inversion, for higher order co/cross polarized scattered field coefficients.

\section{FORMULATION}

The scattering geometry is presented in Figure 1 for a system of infinitely perfect electromagnetic conducting elliptic cylinders with arbitrary size and orientation. Cylinder one is positioned at $\mathrm{o}_{1}$ while the other cylinder is positioned at the point $(\mathrm{d}, \gamma)$. The radii $\mathrm{a}_{1}$ and $\mathrm{a}_{2}$ present the major axes of the cylinders while the minor axes are presented by $b_{1}$ and $b_{2}$. Each cylinder local coordinate system makes an angle, $\alpha_{1}$ for cylinder one while $\alpha_{2}$ for cylinder two, with its global coordinate system.

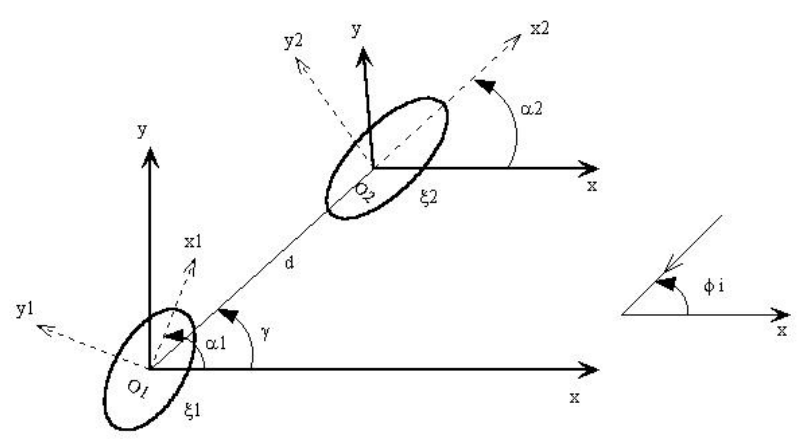

Figure1 Geometry of two PECM elliptic cylinders.

Consider an electromagnetic plane wave incoming towards a system of PEMC elliptic cylinders at an angle $\varphi_{i n c}$ with regard to the $x$ axis, as shown in Figure 1. The electric field component of the TM polarization is given by

$$
E_{z}^{i n c}=E_{0} e^{j k \rho \cos \left(\varphi-\varphi_{i n c}\right)}
$$

where $k$ is the wave number in the region exterior to the elliptic cylinders. The incoming fields towards the system of two cylinders may be expressed as [6] 


$$
\begin{aligned}
& E_{i z}^{i n c}=\sum_{m=0}^{\infty} A_{\text {iem }} R_{\text {em }}^{(1)}\left(c_{i}, \xi_{i}\right) S_{e m}\left(c_{i}, \eta_{i}\right)+ \\
& \sum_{m=1}^{\infty} A_{\text {iom }} R_{o m}^{(1)}\left(c_{i}, \xi_{i}\right) S_{\text {om }}\left(c_{i}, \eta_{i}\right) \\
& H_{i v}^{i n c}=\frac{1}{j k z h}\left[\begin{array}{l}
\left.\sum_{m=0}^{\infty} A_{\text {iem }} R_{\text {em }}^{(1)^{\prime}}\left(c_{i}, \xi_{i}\right) S_{\text {em }}\left(c_{i}, \eta_{i}\right)+\right] \\
\sum_{m=1}^{\infty} A_{\text {iom }} R_{\text {om }}^{(1)^{\prime}}\left(c_{i}, \xi_{i}\right) S_{\text {om }}\left(c_{i}, \eta_{i}\right)
\end{array}\right]
\end{aligned}
$$

where $i=1$ or 2 and the incident field coefficients are

$$
\begin{aligned}
& A_{\substack{1 e m \\
o m}}=E_{0} j^{m} \frac{\sqrt{8 \pi}}{N_{\substack{e m \\
o m}}\left(c_{1}\right)} S_{\substack{e m \\
o m}}\left(c_{1}, \cos \varphi_{i n c}^{1}\right) \\
& A_{2 e m}=E_{0} j^{m} \frac{\sqrt{8 \pi}}{N_{e m}\left(c_{2}\right)} S_{o m}\left(c_{2}, \cos \varphi_{i}^{2}\right) e^{j k d \cos \left(\gamma-\varphi_{i n c}\right)} \\
& N_{\substack{e m \\
o m}}\left(c_{i}\right)=\int_{0}^{2 \pi}\left[S_{\substack{e m \\
o m}}\left(c_{i}, \eta_{i}\right)\right]^{2} d v \\
& \varphi_{i n c}^{1}=\varphi_{i n c}-\alpha_{1}, \varphi_{i n c}^{2}=\varphi_{i n c}-\alpha_{2}
\end{aligned}
$$

and $c_{1}=k F_{1}, c_{2}=k F_{2}, h=F \sqrt{\cosh ^{2} u-\cos ^{2} v}, Z$ is the impedance in the surrounding region to the cylinders, the prime refers to the derivative with respect to $u, S_{e m}$ and $S_{o m}$ are angular Mathieu functions, even and odd with order $m$, respectively, $R_{e m}^{(1)}$ and $R_{o m}^{(1)}$ are the radial Mathieu functions of the first kind, even and odd, and $N_{e m}, N_{o m}$ are normalized functions of even and odd type.

The scattered co/cross electric and magnetic fields from the PEMC elliptic cylinders can be presented in terms of Mathieu functions as

$$
\begin{aligned}
& E_{i z}^{s}=\sum_{m=0}^{\infty} B_{i e m} R_{e m}^{(4)}\left(c_{i}, \xi_{i}\right) S_{e m}\left(c_{i}, \eta_{i}\right)+ \\
& \sum_{m=1}^{\infty} B_{i o m} R_{o m}^{(4)}\left(c_{i}, \xi_{i}\right) S_{o m}\left(c_{i}, \eta_{i}\right) \\
& H_{i v}^{s}=\frac{1}{j k Z h}\left[\begin{array}{l}
\left.\sum_{m=0}^{\infty} B_{i e m} R_{e m}^{(4))^{\prime}}\left(c_{i}, \xi_{i}\right) S_{e m}\left(c_{i}, \eta_{i}\right)+\right] \\
\sum_{m=1}^{\infty} B_{\text {iom }} R_{o m}^{(4))^{\prime}}\left(c_{i}, \xi_{i}\right) S_{o m}\left(c_{i}, \eta_{i}\right)
\end{array}\right]
\end{aligned}
$$

$$
\begin{aligned}
& H_{i z}^{s}=\frac{j}{Z}\left[\begin{array}{l}
\left.\sum_{m=0}^{\infty} C_{i e m} R_{e m}^{(4)}\left(c_{i}, \xi_{i}\right) S_{e m}\left(c_{i}, \eta_{i}\right)+\right] \\
\sum_{m=1}^{\infty} C_{i o m} R_{o m}^{(4)}\left(c_{i}, \xi_{i}\right) S_{o m}\left(c_{i}, \eta_{i}\right)
\end{array}\right] \\
& E_{i v}^{s}=-\frac{1}{k h}\left[\begin{array}{l}
\sum_{m=0}^{\infty} C_{i e m} R_{e m}^{(4)^{\prime}}\left(c_{i}, \xi_{i}\right) S_{e m}\left(c_{i}, \eta_{i}\right)+ \\
\sum_{m=1}^{\infty} C_{i o m} R_{o m}^{(4)^{\prime}}\left(c_{i}, \xi_{i}\right) S_{o m}\left(c_{i}, \eta_{i}\right)
\end{array}\right]
\end{aligned}
$$

where $B_{i e m}, C_{i e m}, B_{i o m}$, and $C_{i o m}$ are the unknown even and odd scattered field expansion coefficients, and $R_{e m}^{(4)}$ and $R_{o m}^{(4)}$ are Mathieu functions, even and odd, of the fourth kind.

Excitation of each cylinder by the incoming plane wave alone generates the first iteration scattered field. The unknown expansion coefficients are obtained by imposing the boundary condition at the surface of the PEMC elliptic cylinders.

For ith cylinder, this can be expressed mathematically, for the co polarized incident fields [9], as

$$
\begin{aligned}
& {\left[H_{i z}^{s 1}+M\left(E_{i z}^{i n c}+E_{i z}^{s 1}\right)\right]_{\xi=\xi_{i}}=0} \\
& {\left[H_{i v}^{i n c}+H_{i v}^{s 1}+M E_{i v}^{s 1}\right]_{\xi=\xi i_{i}}=0}
\end{aligned}
$$

where $M$ is the PEMC admittance. Putting equations (2-11) into equations (12-13) and using orthogonality properties of the angular Mathieu function, we extract the first order co/cross polarized scattered field coefficients as

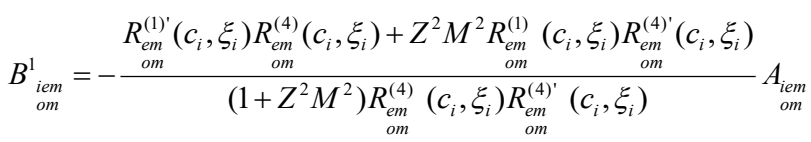

$$
\begin{aligned}
& C_{\substack{i e m \\
o m}}^{1}=j M Z \frac{R_{e m}^{(4))^{\prime}}\left(c_{i}, \xi_{i}\right) R_{e m}^{(1)}\left(c_{i}, \xi_{i}\right)-R_{\substack{e m \\
o m}}^{(1))^{\prime}}\left(c_{i}, \xi_{i}\right) R_{\substack{e m \\
o n}}^{(4)}\left(c_{i}, \xi_{i}\right)}{\left(1+Z^{2} M^{2}\right) R_{e m}^{(4)}\left(c_{i}, \xi_{i}\right) R_{\substack{e m \\
o m}}^{(4)^{\prime}}\left(c_{i}, \xi_{i}\right)} A_{\substack{i e m \\
o m}}
\end{aligned}
$$

The above expressions may be put in matrix form as

$$
\left[\begin{array}{c}
B_{i e m}^{1} \\
o m \\
C_{i e m}^{1} \\
o m
\end{array}\right]=\left[\begin{array}{cc}
Q_{e m m}^{i i} & 0 \\
o m n & \\
0 & Q_{e n m}^{i i c} \\
o m n
\end{array}\right]\left[\begin{array}{c}
A_{i e m} \\
o m \\
A_{i e m} \\
o m
\end{array}\right]
$$

where 


$$
Q_{\substack{\text { enm } \\ \text { omn }}}^{i i}=-\frac{R_{\text {om }}^{(1)^{\prime}}\left(c_{i}, \xi_{i}\right) R_{e m}^{(4)}\left(c_{i}, \xi_{i}\right)+Z^{2} M^{2} R_{e m}^{(1)}\left(c_{i}, \xi_{i}\right) R_{\text {om }}^{(4))^{\prime}}\left(c_{i}, \xi_{i}\right)}{\left(1+Z^{2} M^{2}\right) R_{\text {om }}^{(4)}\left(c_{i}, \xi_{i}\right) R_{\text {om }}^{(4)^{\prime}}\left(c_{i}, \xi_{i}\right)}
$$

$=0, n \neq m$

$$
\begin{aligned}
& R_{e m}^{(4)^{\prime}}\left(c_{i}, \xi_{i}\right) R_{e m}^{(1)}\left(c_{i}, \xi_{i}\right)-R_{e m}^{(1)^{\prime}}\left(c_{i}, \xi_{i}\right) R_{e m}^{(4)}\left(c_{i}, \xi_{i}\right) \\
& Q_{\substack{\text { enm } \\
\text { omn }}}^{i i c}=j M Z \frac{\text { om }}{\left(1+Z^{2} M^{2}\right) R_{\substack{\text { om } \\
\text { om }}}^{(4)}\left(c_{i}, \xi_{i}\right) R_{\text {em }}^{(4)^{\prime}}\left(c_{i}, \xi_{i}\right)} \\
& =0, n \neq m
\end{aligned}
$$

The second iteration scattered co/cross polarized fields are generated from the excitation of cylinders by the first iteration scattered co/cross polarized fields due to the initial incoming fields.

The unknown scattered expansion coefficients may be achieved by forcing the boundary conditions at the surface of the PEMC elliptic cylinders.

For the first cylinder, this can be expressed mathematically due to the co polarization scattered fields of cylinder two incoming field towards the first cylinder as

$$
\begin{aligned}
& {\left[H_{1 z}^{s 2}+M\left(E_{2 z}^{s 1}+E_{1 z}^{s 2}\right)\right]_{\xi=\xi_{1}}=0} \\
& {\left[H_{2 v}^{s 1}+H_{1 v}^{s 2}+M E_{1 v}^{s 2}\right]_{\xi=\xi_{1}}=0}
\end{aligned}
$$

Putting the appropriate expressions in equations (19) and (20), and expressing the first iteration scattered fields from cylinder two in terms of the appropriate system of cylinder one by using the addition theorem for the Mathieu functions [4,5], i.e.,

$$
\begin{gathered}
\underset{\text { om }}{R_{\text {om }}^{(4)}\left(c_{2}, \xi_{2}\right)} \underset{\substack{\text { om } \\
\text { om }}}{ }\left(c_{2}, \eta_{2}\right)=\sum_{l=0}^{\infty} \underset{\text { olm }}{W E_{\text {olm }}^{2 \rightarrow 1}} R_{e l}^{(1)}\left(c_{1}, \xi_{1}\right) S_{e l}\left(c_{1}, \eta_{1}\right) \\
+\sum_{l=1}^{\infty} W O_{\substack{\text { olm } \\
\text { olm }}}^{2 \rightarrow 1} R_{o l}^{(1)}\left(c_{1}, \xi_{1}\right) S_{o l}\left(c_{1}, \eta_{1}\right)
\end{gathered}
$$

where

$$
\begin{aligned}
& \underset{\substack{\text { elm } \\
\text { olm }}}{2 \rightarrow 1}=\frac{\pi j^{l-m}}{N_{e_{1}}\left(c_{1}\right)}=\sum_{i=0}^{\infty} \sum_{p=0}^{\infty}(-j)^{i+p} D_{\substack{e i \\
o i}}^{m}\left(c_{2}\right) D_{e p}^{l}\left(c_{1}\right) \mathrm{X}_{\substack{e i p \\
o i p}}^{2 \quad 1}
\end{aligned}
$$

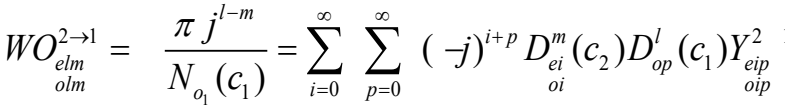

and

$$
\underset{\substack{\text { eip } \\
\text { oip }}}{\mathrm{X}^{\rightarrow 1}}=H_{p-i}^{(2)}(k d)\left[\begin{array}{l}
\cos \Psi^{-} \\
\sin \Psi^{-}
\end{array}\right]+(1)^{i} H_{p+i}^{(2)}(k d)\left[\begin{array}{l}
\cos \Psi^{+} \\
\sin \Psi^{+}
\end{array}\right]
$$

$$
\underset{\substack{\text { eip } \\
\text { oip }}}{2 \rightarrow 1}=H_{p-i}^{(2)}(k d)\left[\begin{array}{l}
\sin \Psi^{-} \\
\cos \Psi^{-}
\end{array}\right]-(-1)^{i} H_{p+i}^{(2)}(k d)\left[\begin{array}{l}
\sin \Psi^{+} \\
\cos \Psi^{+}
\end{array}\right]
$$

with

$$
\Psi^{+}=i \Psi_{21}+p \Psi_{12}, \quad \Psi^{-}=i \Psi_{21}-p \Psi_{12}
$$

where $\Psi_{12}$ and $\Psi_{21}$ are measured from the local $\mathrm{x}$ axis of each cylinder to the distance between the cylinders, $H_{p+i}^{(2)}(k d)$ is the Hankel function of the second kind with argument $k$, and $D_{e j}^{n}$ and $D_{o j}^{n}$ are the Fourier coefficients of the Mathieu functions.

Apply the orthogonality properties of the angular Mathieu functions yield the second order co/cross polarized scattered field coefficients as

$$
\begin{aligned}
& B_{1 e m}^{2}=-\frac{R_{e m}^{(1)^{\prime}}\left(c_{1}, \xi_{1}\right) R_{e m}^{(4)}\left(c_{1}, \xi_{1}\right)+Z^{2} M^{2} R_{e m}^{(1)}\left(c_{1}, \xi_{1}\right) R_{e m}^{(4)^{\prime}}\left(c_{1}, \xi_{1}\right)}{\left(1+Z^{2} M^{2}\right) R_{e m}^{(4)}\left(c_{1}, \xi_{1}\right) R_{e m}^{(4)^{\prime}}\left(c_{1}, \xi_{1}\right)} \\
& {\left[\sum_{l=0}^{\infty} B_{2 e l}^{1} W E_{e l m}^{2 \rightarrow 1}+\sum_{l=0}^{\infty} B_{2 o l}^{1} W E_{\text {olm }}^{2 \rightarrow 1}\right]} \\
& C_{1 e m}^{2}=j M Z \frac{R_{e m}^{(4)^{\prime}}\left(c_{1}, \xi_{1}\right) R_{e m}^{(1)}\left(c_{1}, \xi_{1}\right)-R_{e m}^{(1)^{\prime}}\left(c_{1}, \xi_{1}\right) R_{e m}^{(4)}\left(c_{1}, \xi_{1}\right)}{\left(1+Z^{2} M^{2}\right) R_{e m}^{(4)}\left(c_{1}, \xi_{1}\right) R_{e m}^{(4)^{\prime}}\left(c_{1}, \xi_{1}\right)} \\
& {\left[\sum_{l=0}^{\infty} B_{2 e l}^{1} W E_{e l m}^{2 \rightarrow 1}+\sum_{l=0}^{\infty} B_{2 o l}^{1} W E_{o l m}^{2 \rightarrow 1}\right]}
\end{aligned}
$$

while the odd co and cross first order scattered field coefficients may be obtained from equations (27) and (28). This yield

$B_{1 o m}^{2}=-\frac{R_{o m}^{(1)^{\prime}}\left(c_{1}, \xi_{1}\right) R_{e m}^{(4)}\left(c_{1}, \xi_{1}\right)+Z^{2} M^{2} R_{e m}^{(1)}\left(c_{1}, \xi_{1}\right) R_{e m}^{(4) '}\left(c_{1}, \xi_{1}\right)}{\left(1+Z^{2} M^{2}\right) R_{e m}^{(4)}\left(c_{1}, \xi_{1}\right) R_{e m}^{(4) '}\left(c_{1}, \xi_{1}\right)}$

$\left[\sum_{l=0}^{\infty} B_{2 e l}^{1} W O_{e l m}^{2 \rightarrow 1}+\sum_{l=0}^{\infty} B_{2 o l}^{1} W O_{o l m}^{2 \rightarrow 1}\right]$

$C_{1 o m}^{2}=j M Z \frac{R_{o m}^{(4)^{\prime}}\left(c_{1}, \xi_{1}\right) R_{o m}^{(1)}\left(c_{1}, \xi_{1}\right)-R_{o m}^{(1)^{\prime}}\left(c_{1}, \xi_{1}\right) R_{o m}^{(4)}\left(c_{1}, \xi_{1}\right)}{\left(1+Z^{2} M^{2}\right) R_{o m}^{(4)}\left(c_{1}, \xi_{1}\right) R_{o m}^{(4)^{\prime}}\left(c_{1}, \xi_{1}\right)}$

$\left[\sum_{l=0}^{\infty} B_{2 e l}^{1} W O_{e l m}^{2 \rightarrow 1}+\sum_{l=0}^{\infty} B_{2 o l}^{1} W o_{o l m}^{2 \rightarrow 1}\right]$

(30)

Equations (27)-(30) may be written in matrix form as

$\left[\begin{array}{c}B_{1 \mathrm{em}}^{2} \\ B_{1 \mathrm{om}}^{2}\end{array}\right]=\left[\begin{array}{cc}Q_{\text {enm }}^{11} & 0 \\ 0 & Q_{\text {onm }}^{11}\end{array}\right]\left[\begin{array}{ll}Q_{\text {eenm }}^{12} & Q_{\text {eonm }}^{12} \\ Q_{\text {oenm }}^{12} & Q_{\text {oonm }}^{12}\end{array}\right]\left[\begin{array}{l}B_{2 \text { em }}^{1} \\ B_{2 \text { om }}^{1}\end{array}\right]$ 
$\left[\begin{array}{c}C_{1 e m}^{2} \\ C_{1 o m}^{2}\end{array}\right]=\left[\begin{array}{cc}Q_{\text {enm }}^{11 c} & 0 \\ 0 & Q_{\text {onm }}^{11 c}\end{array}\right]\left[\begin{array}{ll}Q_{\text {eenm }}^{12} & Q_{\text {eonm }}^{12} \\ Q_{\text {oenm }}^{12} & Q_{\text {oonm }}^{12}\end{array}\right]\left[\begin{array}{c}B_{2 e m}^{1} \\ B_{2 o m}^{1}\end{array}\right]$

where $\quad Q_{\text {eenm }}^{12}=W E_{\text {enm }}^{2 \rightarrow 1}, \quad Q_{\text {eonm }}^{12}=W E_{\text {onm }}^{2 \rightarrow 1}$

$Q_{\text {oenm }}^{12}=W O_{\text {enm }}^{2 \rightarrow 1}$ and $Q_{\text {oonm }}^{12}=W O_{\text {onm }}^{2 \rightarrow 1}$.

Finally, for the first cylinder, the scattered field coefficients due to the cross polarization scattered fields of the second cylinder incident on the first cylinder may be written as

$\left[\begin{array}{c}D_{\text {lem }}^{2} \\ D_{1 o m}^{2}\end{array}\right]=\left[\begin{array}{cc}Q_{\text {enm }}^{11} & 0 \\ 0 & Q_{\text {onm }}^{11}\end{array}\right]\left[\begin{array}{ll}Q_{\text {eenm }}^{12} & Q_{\text {eonm }}^{12} \\ Q_{\text {oenm }}^{12} & Q_{\text {oonm }}^{12}\end{array}\right]\left[\begin{array}{l}C_{2 e m}^{1} \\ C_{2 o m}^{1}\end{array}\right]$

(33)

$\left[\begin{array}{c}G_{1 e m}^{2} \\ G_{1 o m}^{2}\end{array}\right]=\left[\begin{array}{cc}Q_{\text {enm }}^{11 c} & 0 \\ 0 & Q_{\text {onm }}^{11 c}\end{array}\right]\left[\begin{array}{ll}Q_{\text {eenm }}^{12} & Q_{\text {eonm }}^{12} \\ Q_{\text {oenm }}^{12} & Q_{\text {oonm }}^{12}\end{array}\right]\left[\begin{array}{c}C_{2 e m}^{1} \\ C_{2 o m}^{1}\end{array}\right]$

The expression for the higher order scattered co/cross polarized field coefficients for a system of cylinders may be written following the previous procedures.

The total scattered field from the cylinders due to higher order scattered field can be written as

$E_{z}^{s}=\left(\frac{j}{k \rho}\right)^{0.5} e^{-j k \rho}\left\{\sum_{k=1,2, \ldots}\left\{\sum_{\substack{m=0 \\ m^{m}}}^{\infty} B_{\substack{k m \\ o m}}^{k} S_{\substack{e m \\ \text { om }}}\left(c_{1}, \cos \left(\varphi-\alpha_{1}\right)\right)\right.\right.$

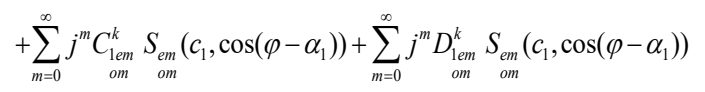

$\sum_{m=0}^{\infty} j^{m} G_{1 m}^{k} S_{\substack{e m \\ o m}}^{k}\left(c_{1}, \cos \left(\varphi-\alpha_{1}\right)\right)+$

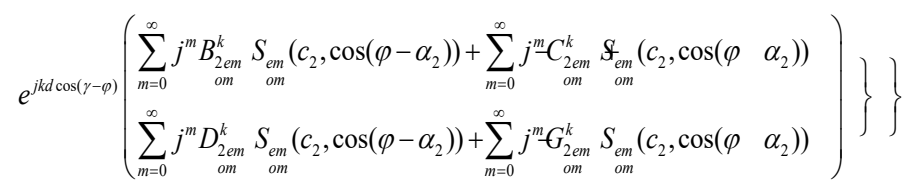

For the TM polarization case, the echo is defined as

$$
\sigma_{T M}=2 \pi \rho \lim _{\rho \rightarrow \infty} \frac{\left|E_{z}^{s}\right|^{2}}{\left|E_{z}^{i}\right|^{2}}
$$

\section{CONCLUSION}

Rigorous solution to the problem of field scattered due to a plane electromagnetic wave incoming on a system of PEMC elliptic cylinders was presented. Translation addition theorem was used in order to satisfy iteratively the boundary conditions at the cylinders surface. The obtained system of equations was written in matrix form and the scattered field coefficients for co and cross polarized fields were obtained without matrix inversion.

\section{ACKNOWLEDGEMENT}

Authors would like to acknowledge the help provided by the University of Sharjah, Sharjah, UAE.

\section{REFERENCES}

[1] A. Sebak, "Electromagnetic scattering by two parallel dielectric elliptic cylinders," IEEE Trans. Antennas Propagat. Vol. AP-42, pp. 1521-1527, 1994.

[2] A-K. Hamid and M.I.Hussein, "Iterative solution to the electromagnetic plane wave scattering by two parallel conducting elliptic cylinders, " J. of Electromagnetic Waves and Applications, Vol. 17, pp. 813-828, 2003.

[3] A-K. Hamid, "Iterative Solution to the TM Scattering by Two Infinitley Long Lossy Dielelctric Elliptic Cylinders," J. of Electromagnetic Waves and Applications, vol. 18, pp. 529-546, 2004.

[4] H. Ragheb and M. Hamid, "Scattering by N parallel conducting circular cylinders," Int. J. of Electronics, vol. 59, pp. 407-421, 1985.

[5] A.Z. Elsherbeni and M. Hamid, "Scattering by parallel conducting circular cylinders," IEEE Trans. Antennas Propagat. Vol. AP-35, pp. 335-358, 1987.

[6] S. Ahmed and Q.A. Naqvi, "Electromagntic scattering from parallel PEMC cylinders of circular Cross sections using iterative procedure," . J. of Electromagnetic Waves and Applications, vol. 22, pp. 987-1003, 2008.

[7] A-K. Hamid, I.R. Ciric, and M. Hamid, "Iterative solution of the scattering by an arbitrary configuration of conducting or dielectric spheres," IEE Proc. Part H, vol. 138, pp. 565-572, 1991.

[8] A-K. Hamid and F.R. Cooray, "Scattering by a perfect electromagnetic conducting elliptic cylinder", Progress In Electromagnetic Research, PIER, Vol. 10, 59-67, 2009. 